\title{
Polymyalgia rheumatica and vertebral fractures: a 1-year pilot controlled study
}

\author{
Luigi Calvo $\cdot$ Giovanni Pistone $\cdot$ Sabrina Arnone $\cdot$ Daniela Colomba \\ Salvatore Amico $\cdot$ Antonella Giacalone $\cdot$ Pietra Vitale $\cdot$ Calogero Nicosia $\cdot$ \\ Eliana Barone · Rosario Scaglione · Giuseppe Licata $\cdot$ Salvatore Corrao
}

Received: 9 October 2009 / Accepted: 27 February 2010 / Published online: 19 March 2010

(C) Springer-Verlag 2010

\begin{abstract}
No data exist about the possibility that vertebral fracture in PMR patients could be independent of steroid therapy. For this reason, we aimed to investigate this topic by a case cohort study with a 1-year follow-up for each patient. We selected ten consecutive patients who experienced vertebral fractures (VF-group) during the first month of 1-year follow-up period and without any other significant associated condition. As a control group we studied ten control patients, without vertebral fractures and with a follow-up of 1 year, randomly selected among a larger group of patients affected by polymyalgia rheumatica. The following data were analysed: eritrosedimention rate (ESR), visual analogical scale score (VAS), methyprednisolone daily dosage. Each patient had been monthly evaluated by the aforementioned clinical and laboratoristic parameters during the 1-year follow-up period. The VFgroup resulted with a higher and statistically significant median corticosteroid 12-month total dosage [mean
\end{abstract}

L. Calvo $\cdot$ D. Colomba $\cdot$ S. Amico $\cdot$ E. Barone

R. Scaglione $\cdot$ G. Licata $\cdot$ S. Corrao $(\bowtie)$

Dipartimento Biomedico di Medicina Interna e Specialistica,

Università di Palermo, Piazza delle Cliniche 2,

90127 Palermo, Italy

e-mail: s.corrao@tiscali.it

G. Pistone $\cdot$ P. Vitale $\cdot$ C. Nicosia

Outpatient Rheumatologic Clinic,

National Relevance Hospital Trust "Civico e Benfratelli,

G. Di Cristina, M. Ascoli”, Palermo, Italy

S. Arnone $\cdot$ A. Giacalone

Unit of Clinical Methodology,

Epidemiology and Statistics,

National Relevance Hospital Trust "Civico e Benfratelli,

G. Di Cristina, M. Ascoli”, Palermo, Italy
$3,480 \mathrm{mg}(95 \% \mathrm{CI} 2,805-3,030)$ vs. $2,760 \mathrm{mg}(2,666.25-$ $3,247.5)$ ]. The VF-group had statistically significant higher ESR and VAS AUC when compared to control group (median ESR AUC, 484.75 vs. 288.25; $P=0.0001$; median VAS AUC, 70.75 vs. $43.5 P<0.0001$ ); ESR at the baseline (cut-off $>80 \mathrm{~mm}$ ) showed a specificity of $90 \%$ (95\%CI $56-$ $100)$ and sensitivity of $70 \%$ (95\%CI 35-93). VAS difference from first to second month (cut-off $\leq 3$ ) showed a specificity of $90 \%$ (95\% CI 56-100) and sensitivity of $80 \%$ (95\% CI 44-97). Our results point out that vertebral fracture might be predicted from commonly used laboratory (ESR) and clinical (VAS) findings.

Keywords Polymyalgia rheumatica - Vertebral fractures . Osteoporosis $\cdot$ Controlled clinical trial

\section{Introduction}

Polymyalgia Rheumatica (PMR) is an inflammatory condition of unknown cause that is characterized by an aching and morning stiffness in the shoulder and pelvic girdles other than the same kind of symptoms in the cervical region.

Well-documented data are available from literature about PMR and osteoporosis (1-3). In particular, the beneficial effects of corticosteroid therapy in the treatment of PMR may be offset by the occurrence of corticosteroidrelated osteoporosis. So, several studies have addressed the question of what dose of corticosteroids does not determine deleterious effect on bone density: the results of these studies prove that this dose is uncertain (4). On the other hand, in our knowledge, there are no data about both the frequency of vertebral fractures and related clinical features in recently diagnosed PMR patients. Moreover, no data 
exist about the possibility that vertebral fracture in PMR patients could be independent of steroid therapy.

For this reason, we aimed to investigate this last topic by a case cohort study with a 1-year follow-up for each polymyalgia rheumatica patient who had experienced a vertebral fracture within the first month after the diagnosis.

\section{Methods}

We recruited ten consecutive patients who experienced vertebral fractures (VF-group) between during a previous 2-year period (2006-2007 years) affected by first diagnosed polymyalgia rheumatica, followed-up for 1 year, and without any other significant associated condition. All the fractures were found out in acute symptomatic patients and had occurred during the first month after the diagnosis. As a control group, we studied ten control patients, without vertebral fractures and with a follow-up of 1 year, randomly selected among a larger group of patients affected by PMR. Randomization was performed by links in order to obtain a matched control group from some relevant variables (age, BMI, gender, comorbidities and drug therapy).

All the patients were referred to the Rheumatologic outpatient clinic of our Hospital trust, and all met the ACR criteria for diagnosis of PMR $(5,6)$. Moreover, in each patient of both groups PMR was diagnosed within 1 month from the onset of symptoms.

Each patient underwent an X-ray at baseline, after 12 months, and when experienced an acute pain along spine. The vertebral fractures were first evaluated by X-ray after an acute aching episode and confirmed by axial computerized tomography. We analysed the following data: eritrosedimention rate (ESR), visual analogical scale score (VAS) for pain assessment, methyprednisolone daily dosage. Each patient had been monthly evaluated by the aforementioned clinical and laboratoristic parameters during the 1-year follow-up period. The two groups did not meaningfully differ for gender and age, neither there were meaningful differences for steroidal dosing, ESR, VAS at baseline. Moreover, the two groups did not differ for percentage of patients overweight, hypertensive and diabetic (Table 1). There were no significant differences about drug therapy (antidiabetic and antihypertensive) between the two groups.

\section{Statistical analysis}

The results are presented as mean value \pm standard deviation, median (interquartile range, as lower and upper quartile) and percentages when appropriated. Unpaired $t$ test was used for comparison of means, Mann-Whitney $U$ test for comparison of medians, and Fisher's exact test for comparison of proportions. Area under curve (AUC) of ESR and VAS, from baseline to 12 month control, was computed for comparison between groups. Predictive values (post-test likelihood) with change, prevalence (pretest likelihood), sensitivity, specificity and likelihood ratios with robust confidence intervals were computed from ESR and VAS values for prediction of vertebral fractures. ROC plots and a ROC analysis were performed to individuate best sensitivity and specificity cut-off points. A two-tailed alpha value of $<0.05$ was considered to be significant.

\section{Results}

Study population variables at baseline are shown in Table 1.

Figures 1 and 2 show ESR and VAS trends of the VFgroup compared to the control one during 1-year follow-up.

Although the total amount of corticosteroids during the first 3 months of observation was not significantly different between the two groups, the VF-group resulted with a higher and statistically significant $(P=0.0135)$ median corticosteroid 12-month total dosage [3,480 mg (2,805-3,030) versus $2,760 \mathrm{mg}(2,666.25-3,247.5)]$. The VF-group had statistically significant higher ESR and VAS AUC when compared to control group (median ESR AUC, 484.75 vs.
Table 1 Patient characteristics and variables at baseline: control group versus VF-group

$V F$-group patients with vertebral fractures, ESR eritrosedimention rate, $V A S$ visual analogical scale score for pain assessment

* Statistically significant comparison $(P=0.0403)$

\begin{tabular}{lll}
\hline & Control group & VF-group \\
\hline No. & 10 & 10 \\
Gender (W/M) & $(5 / 5)$ & $(5 / 5)$ \\
Age (years) (mean \pm 1SD) & $75.6 \pm 3.38$ & $76.6 \pm 4.6$ \\
Prednisone dosage at baseline [median (lower-upper quartile)] & $20(20-25)$ & $20(20-25)$ \\
ESR at baseline [median (lower-upper quartile)] & $77(75-80)$ & $86.5(77.5-90)^{*}$ \\
VAS at baseline [median (lower-upper quartile)] & $10(9-10)$ & $10(9-10)$ \\
No. of overweight patients & 8 & 8 \\
No. of hypertensive patients & 4 & 4 \\
No. of diabetic patients & 1 & 2 \\
\hline
\end{tabular}




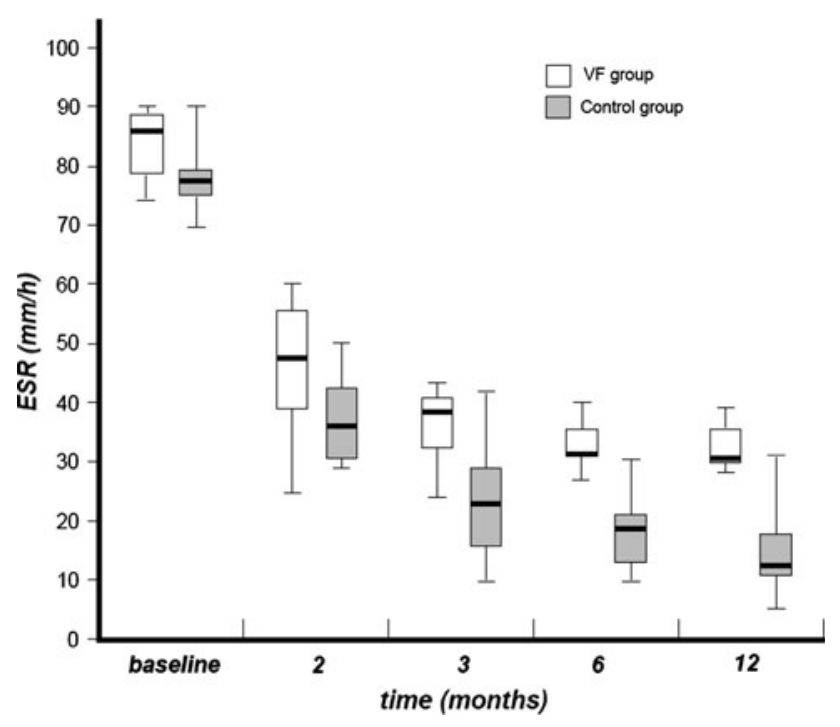

Fig. 1 Box-wisker plot of ESR value year trend. Comparison between VF (vertebral fracture) and control groups (median value: thick line, 1 st and 3rd quartiles values, lower and upper box limits, min and max values, lower and upper wisker limits)

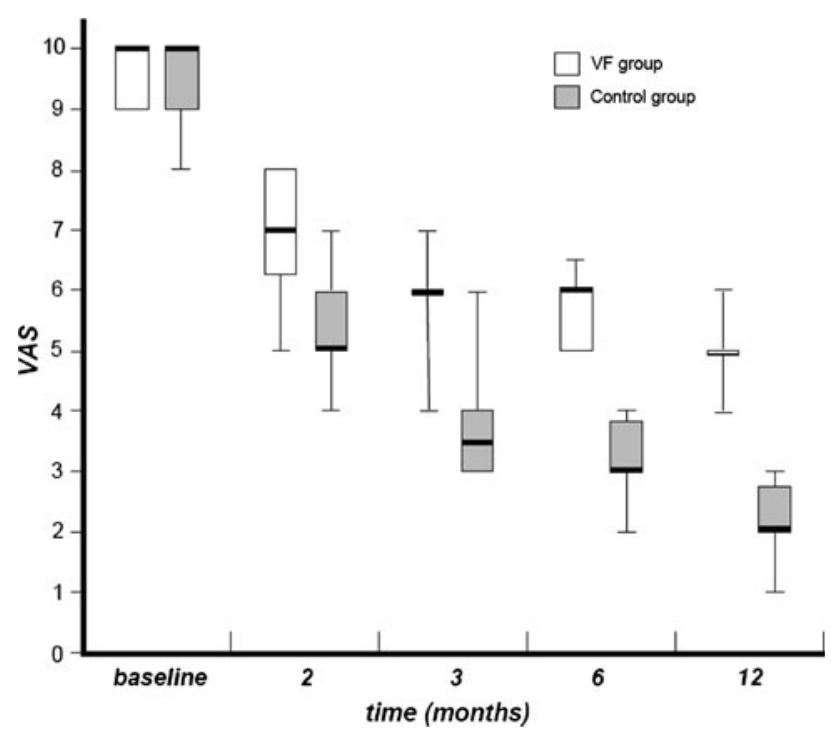

Fig. 2 Box-wisker plot of VAS score year trend. Comparison between VF (vertebral fracture) and control groups (median value: thick line, 1 st and 3rd quartiles values, lower and upper box limits, min and max values, lower and upper wisker limits)

288.25; $P=0.0001 ;$ median VAS AUC, 70.75 vs. 43.5 $P<0.0001)$;

ESR at the baseline (cut-off $>80 \mathrm{~mm}$ ) showed a specificity of 90\% (95\% CI 56-100) and sensitivity of 70\% (95\%CI 35-93). VAS difference from first to second month (cut-off $\leq 3)$ showed a specificity of $90 \%(95 \%$ CI $56-100)$ and sensitivity of $80 \%$ (95\% CI 44-97).

\section{Discussion}

To our knowledge, this study is the first that appraises the incidence of vertebral fractures after the clinical onset of the PMR. Our study deals with a case cohort study about 10 patients affected by PMR with recent vertebral fractures (VF-group) compared to 10 patients affected by PMR without vertebral fractures (NF-group). This is a pilot-study considered both the design and the sample size. However, this kind of design has allowed a strict selection of controls closely matched to the cases for meaningful variables.

So, conclusions of our study and related hypothesis could be sufficiently strong to both elicit interest in scientific community on this topic and consequently draw sound clinical trials. Our results show that ESR was higher in VF-group in comparison with controls $(P=0.0403)$ at baseline. This finding suggests that subjects who will develop vertebral fractures precociously after the onset of PMR could belong to a subgroup of PMR subjects with a higher probability of vertebral fractures independent from steroid osteoporotic bone effects.

In conclusion, our preliminary results point out that vertebral fractures both represent a problem for patients affected by PMR, during the first months after the onset of symptoms, and could be predicted from commonly used laboratory (ESR) and clinical (VAS) findings. Apart from the importance to investigate pathophysiological mechanisms, an effort to understand the importance of our data and the needs for a precocious instrumental evaluation for osteoporosis, in this kind of patients, might be investigated in future studies for better utilizing economic resources.

\section{References}

1. Homik J, Suarez-Almazor ME, Shea B, Cranney A, Wells G, Tugwell P (2000) Calcium and vitamin D for corticosteroid-induced osteoporosis. Cochrane Database Syst Rev (2):CD000952

2. Homik J, Cranney A, Shea B, Tugwell P, Wells G, Adachi R, Suarez-Almazor M (2000) Bisphosphonates for steroid induced osteoporosis. Cochrane Database Syst Rev (2):CD001347

3. Weyand CM, Goronzy JJ (2001) Polymyalgia rheumatica and giant cell arteritis. In: Koopman WJ (ed) Arthritis and allied conditionsa textbook of rheumatology. pp 1784-1798

4. Pearce G, Ryan PF, Delmas Pd, Tabensky DA, Seeman E (1998) The deleterious effects of low-dose corticosteroids on bone density in patients with polymyalgia rheumatica. British $\mathrm{J}$ Rheumatol 37(3):292-299

5. Chuang TY, Hunder GG, Ilstrup DM, Kurland LT (1982) Polymyalgia rheumatica: a 10-year epidemiologic and clinical study. Ann Intern Med 97:672-680

6. Healey LA (1984) Long-term follow-up of polymyalgia rheumatica: evidence for synovitis. Semin Arthritis Rheum 13:322-328 